\title{
Almost Automorphic Solutions to Nonautonomous Stochastic Functional Integrodifferential Equations
}

\author{
Li Xi-liang and Han Yu-liang \\ School of Mathematics and Information Sciences, Shandong Institute of Business and Technology, Yantai 264005, China \\ Correspondence should be addressed to Li Xi-liang; lixiliang@amss.ac.cn
}

Received 28 January 2013; Accepted 7 March 2013

Academic Editor: Zhiming Guo

Copyright ( 2013 L. Xi-liang and H. Yu-liang. This is an open access article distributed under the Creative Commons Attribution License, which permits unrestricted use, distribution, and reproduction in any medium, provided the original work is properly cited.

This paper concerns the square-mean almost automorphic solutions to a class of abstract semilinear nonautonomous functional integrodifferential stochastic evolution equations in real separable Hilbert spaces. Using the so-called "Acquistapace-Terreni" conditions and Banach contraction principle, the existence, uniqueness, and asymptotical stability results of square-mean almost automorphic mild solutions to such stochastic equations are established. As an application, square-mean almost automorphic solution to a concrete nonautonomous integro-differential stochastic evolution equation is analyzed to illustrate our abstract results.

\section{Introduction}

Stochastic differential equations in both finite and infinite dimensions, which are important from the viewpoint of applications since they incorporate natural randomness into the mathematical description of the phenomena and hence provide a more accurate description of it, have received considerable attention. Based on this viewpoint, there has been an increasing interest in extending certain classical deterministic results to stochastic differential equations in recent years. As a good case in point, the existence of almost periodic or pseudo-almost periodic solutions to stochastic evolution equations has been extensively considered in many publications; see [1-8] and the references therein.

Integrodifferential equations are used to describe lots of phenomena arising naturally from many fields such as fluid dynamics, number reactor dynamics, population dynamics, electromagnetic theory, and biological models, most of which cannot be described by classical differential equations, and hence they have attracted more and more attention in recent years; see [1, 9-12] for more details.

Recently, Keck and McKibben [9, 10] proposed a general abstract model for semilinear functional stochastic integrodifferential equations and studied the existence and uniqueness of mild solutions to these equations. Based on their works, the existence and uniqueness of square-mean almost periodic solutions to some functional integrodifferential stochastic evolution equations was carefully investigated in [1] for the autonomous case and in our forthcoming paper for the nonautonomous case. In a very recent paper, as a natural generalization of the notion of square-mean almost periodicity, a new concept of squaremean almost automorphic stochastic process was introduced by $\mathrm{Fu}$ and Liu [13], and the existence results of squaremean almost automorphic mild solutions to some linear and semilinear autonomous stochastic differential equations were formulated, while paper [14] investigated the same issue for nonautonomous stochastic differential equations. Under some suitable assumptions, the authors established in a forthcoming paper the existence and uniqueness of squaremean almost automorphic solutions to a class of autonomous functional integrodifferential stochastic evolution equations.

In this paper, we are concerned with a class of semilinear nonautonomous functional stochastic integrodifferential equations in a real separable Hilbert space in the abstract form:

$$
\begin{aligned}
X^{\prime}(t)= & A(t) X(t)+\int_{-\infty}^{t} C(t-s) G(s, X(s)) d W(s) \\
& +\int_{-\infty}^{t} B(t-s) F_{2}(s, X(s)) d s \\
& +F_{1}(t, X(t)), \quad t \in \mathbb{R}
\end{aligned}
$$


where $A(t): D(A(t)) \quad \subset \quad \mathscr{L}^{2}(\mathbf{P} ; \mathbb{U}) \rightarrow \mathscr{L}^{2}(\mathbf{P} ; \mathbb{1})$ is a family of densely defined closed (possibly unbounded) linear operator satisfying the so-called "Acquistapace-Terreni" conditions introduced in [15], $B$ and $C$ are convolution-type kernels in $\mathscr{L}^{1}(0, \infty)$ and $\mathscr{L}^{2}(0, \infty)$, respectively, satisfying Assumption 3.2 in [16], $W(t)$ is a two-sided standard onedimensional Brownian motion defined on the filtered probability space $\left(\Omega, \mathscr{F}, \mathbf{P}, \mathscr{F}_{t}\right)$, where $\mathscr{F}_{t}=\sigma\{W(u)-W(v) ; u, v \leq$ t\}. Here $F_{1}, F_{2}, G: \mathbb{R} \times \mathscr{L}^{2}(\mathbf{P} ; \mathbb{\square}) \rightarrow \mathscr{L}^{2}(\mathbf{P} ; \mathbb{\square})$ are jointly continuous functions satisfying some additional conditions to be specified later in Section 3.

Motivated by the aforementioned works $[1,13,14]$, we investigate in this paper the existence and uniqueness of square-mean almost automorphic solutions to nonautonomous equation (1). The main tools employed here are Banach contraction principle and an estimate on the Ito integral. The obtained results can be seen as a contribution to this emerging field.

The paper is organized as follows. In Section 2, we review some basic definitions and preliminary facts on square-mean almost automorphic processes which will be used throughout this paper. Section 3 is devoted to establish the existence, uniqueness, and the asymptotical stability of square-mean almost automorphic mild solution to (1). As an illustration of our abstract result, square-mean almost automorphic solution to a concrete nonautonomous integrodifferential stochastic evolution equation is investigated in Section 4.

\section{Preliminaries}

To begin this paper, we recall some primary definitions, notations, lemmas, and technical results which will be used in the sequel. For more details on almost automorphy and stochastic differential equations, the readers are referred to $[13,17-23]$ and the references therein.

Throughout this paper, we assume that $(\mathbb{U},\|\cdot\|)$ is a real separable Hilbert space, $(\Omega, \mathscr{F}, \mathbf{P})$ is a probability space, and $\mathscr{L}^{2}(\mathbf{P} ; \mathbb{U})$ stands for the space of all $\mathbb{\square}$-valued random variables $X$ such that

$$
\mathbf{E}\|X\|^{2}=\int_{\Omega}\|X\|^{2} d \mathbf{P}<\infty
$$

For $X \in \mathscr{L}^{2}(\mathbf{P} ; \mathbb{W})$, let

$$
\|X\|_{2}:=\left(\int_{\Omega}\|X\|^{2} d \mathbf{P}\right)^{1 / 2}
$$

It is routine to check that $\mathscr{L}^{2}(\mathbf{P} ; \mathbb{M})$ is a Banach space equipped with the norm $\|\cdot\|_{2}$.

It is well-known that Brownian motion plays a key role in the construction of stochastic integrals. Throughout this paper, $W(t)$ denotes a two-sided standard one-dimensional Brownian motion defined on the filtered probability space $\left(\Omega, \mathscr{F}, \mathbf{P}, \mathscr{F}_{t}\right)$, where $\mathscr{F}_{t}=\sigma\{W(u)-W(v) ; u, v \leq t\}$.
Definition 1. A standard one-dimensional Brownian motion is a continuous, adapted real-valued stochastic process $(W(t), t \geq 0)$ such that

(i) $W(0)=0$ a.s.;

(ii) $W(t)-W(s)$ is independent of $\mathscr{F}_{s}$ for all $0 \leq s<t$;

(iii) $W(t)-W(s)$ is $N(0, t-s)$ distributed for all $0 \leq s \leq t$.

The following definitions and lemmas concerning squaremean almost automorphic functions can be found in $[13,14]$.

Definition 2. A stochastic process $X: \mathbb{R} \rightarrow \mathscr{L}^{2}(\mathbf{P} ; \mathbb{W})$ is said to be stochastically continuous if

$$
\lim _{t \rightarrow s} \mathbf{E}\|X(t)-X(s)\|^{2}=0 .
$$

Definition 3. A stochastically continuous stochastic process $X: \mathbb{R} \rightarrow \mathscr{L}^{2}(\mathbf{P} ; \mathbb{W})$ is said to be square-mean almost automorphic if for every sequence of real numbers $\left\{s_{n}^{\prime}\right\}$ there exists a subsequence $\left\{s_{n}\right\}$ and a stochastic process $Y: \mathbb{R} \rightarrow$ $\mathscr{L}^{2}(\mathbf{P} ; \mathbb{U})$ such that

$$
\begin{aligned}
& \lim _{n \rightarrow \infty} \mathbf{E}\left\|X\left(t+s_{n}\right)-Y(t)\right\|^{2}=0, \\
& \lim _{n \rightarrow \infty} \mathbf{E}\left\|X\left(t+s_{n}\right)-Y(t)\right\|^{2}=0
\end{aligned}
$$

hold for each $t \in \mathbb{R}$. The collection of all square-mean almost automorphic stochastic processes is denoted by $A A\left(\mathbb{R} ; \mathscr{L}^{2}(\mathbf{P} ; \mathbb{R})\right)$.

Lemma 4. If $X, X_{1}$, and $X_{2}$ are all square-mean almost automorphic stochastic processes, then the following statements hold true:

(i) $X_{1}+X_{2}$ is square-mean almost automorphic;

(ii) $\lambda X$ is square-mean almost automorphic for every scalar $\lambda$;

(iii) There exists a constant $M>0$ such that $\sup _{t \in \mathbb{R}}\|X(t)\|_{2}$ $\leq M$. That is, $X$ is bounded in $\mathscr{L}^{2}(\mathbf{P} ; \mathbb{U})$.

Lemma 5. $A A\left(\mathbb{R} ; \mathscr{L}^{2}(\mathbf{P} ; \mathbb{U})\right)$ is a Banach space when it is equipped with the norm

$$
\|X\|_{\infty}:=\sup _{t \in \mathbb{R}}\|X(t)\|_{2}=\sup _{t \in \mathbb{R}}\left(\mathbf{E}\|X(t)\|^{2}\right)^{1 / 2}
$$

for $X \in A A\left(\mathbb{R} ; \mathscr{L}^{2}(\mathbf{P} ; \mathbb{H})\right)$.

Definition 6. A jointly continuous function $F: \mathbb{R} \times$

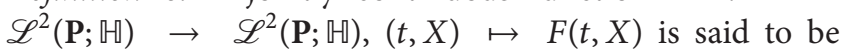
square-mean almost automorphic in $t \in \mathbb{R}$ for each $X \in$

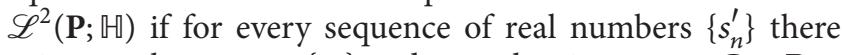
exists a subsequence $\left\{s_{n}\right\}$ and a stochastic process $G: \mathbb{R} \times$ $\mathscr{L}^{2}(\mathbf{P} ; \mathbb{U}) \rightarrow \mathscr{L}^{2}(\mathbf{P} ; \mathbb{U})$ such that

$$
\begin{gathered}
\lim _{n \rightarrow \infty} \mathbf{E}\left\|F\left(t+s_{n}, X\right)-G(t, X)\right\|^{2}=0, \\
\lim _{n \rightarrow \infty} \mathbf{E}\left\|G\left(t-s_{n}, X\right)-F(t, X)\right\|^{2}=0
\end{gathered}
$$

hold for each $t \in \mathbb{R}$ and each $X \in \mathscr{L}^{2}(\mathbf{P} ; \mathbb{U})$. 
Lemma 7. Let $f: \mathbb{R} \times \mathscr{L}^{2}(\mathbf{P} ; \mathbb{U}) \rightarrow \mathscr{L}^{2}(\mathbf{P} ; \mathbb{U}),(t, X) \mapsto$ $f(t, X)$ be square-mean almost automorphic in $t \in \mathbb{R}$ for each $X \in \mathscr{L}^{2}(\mathbf{P} ; \mathbb{1})$, and assume that $f$ satisfies a Lipschitz condition in the following sense:

$$
\mathbf{E}\|f(t, \varphi)-f(t, \psi)\|^{2} \leq L \mathbf{E}\|\varphi-\psi\|^{2}
$$

for all $\varphi, \psi \in \mathscr{L}^{2}(\mathbf{P} ; \mathbb{\square})$ and each $t \in \mathbb{R}$, where $L>0$ is independent of $t$. Then for any square-mean almost automorphic stochastic process $X: \mathbb{R} \rightarrow \mathscr{L}^{2}(\mathbf{P} ; \mathbb{U})$, the stochastic process $F: \mathbb{R} \rightarrow \mathscr{L}^{2}(\mathbf{P} ; \mathbb{\square})$ given by $F(t):=f(t, X(t))$ is square-mean almost automorphic.

The Acquistapace-Terreni conditions (ATCs, for short) play an important role in the study of nonautonomous stochastic differential equations. We state it below for the readers' convenience.

ATCs. There exist constants $\lambda_{0} \geq 0, \theta \in(\pi / 2, \pi), L, K \geq 0$, and $\alpha, \beta \in(0,1]$ with $\alpha+\beta>1$ such that

$$
\begin{gathered}
\Sigma_{\theta} \cup\{0\} \subset \rho\left(A(t)-\lambda_{0}\right), \\
\left\|R\left(\lambda, A(t)-\lambda_{0}\right)\right\| \leq \frac{K}{1+|\lambda|}, \\
\|\left(A(t)-\lambda_{0}\right) R\left(\lambda, A(t)-\lambda_{0}\right) \\
\times\left[R\left(\lambda_{0}, A(t)\right)-R\left(\lambda_{0}, A(s)\right)\right] \| \leq L|t-s|^{\alpha}|\lambda|^{\beta}
\end{gathered}
$$

for $t, s \in \mathbb{R}, \lambda \in \Sigma_{\theta}:=\{\lambda \in \mathbb{C}-\{0\}:|\arg \lambda| \leq \theta\}$.

The following lemma can be found in $[15,24,25]$.

Lemma 8. Suppose that the ATCs are satisfied, and then there exists a unique evolution family $\{U(t, s)\}_{-\infty<s \leq t<\infty}$ on $\mathscr{L}^{2}(\mathbf{P} ; \mathbb{U})$, which governs the linear part of $(1)$.

\section{Main Results}

In this section, we investigate the existence and uniqueness of square-mean almost automorphic solution to the nonautonomous stochastic integrodifferential evolution equation (1). The following assumptions are imposed on (1) which will be assumed throughout the manuscript.

(H1) The operator $A(t): D(A(t)) \quad \subset \quad \mathscr{L}^{2}(\mathbf{P} ; \mathbb{M}) \quad \rightarrow$ $\mathscr{L}^{2}(\mathbf{P} ; \llbracket)$ is a family of densely defined closed linear operators satisfying the ATCs, and the generated evolution family $U(t, s)$ is uniformly exponentially stable; that is, there exist constants $M \geq 1$ and $\delta>0$ such that

$$
\|U(t, s)\| \leq M e^{-\delta(t-s)}, \quad \forall t \geq s .
$$

(H2) The evolution family $\{U(t, s), t \geq s\}$ generated by $A(t)$ satisfies the following condition: from every sequence of real numbers $\left\{s_{n}^{\prime}\right\}_{n \in \mathbb{N}}$, we can extract a subsequence $\left\{s_{n}\right\}_{n \in \mathbb{N}}$ such that, for any $\varepsilon>0$, there exists an $N \in \mathbb{N}$ such that $n>N$ implies that

$$
\begin{gathered}
\left\|U\left(t+s_{n}, s+s_{n}\right)-U(t, s)\right\| \leq \varepsilon e^{-\delta(t-s)}, \\
\left\|U\left(t-s_{n}, s-s_{n}\right)-U(t, s)\right\| \leq \varepsilon e^{-\delta(t-s)}
\end{gathered}
$$

for all $t \geq s$, where $\delta>0$ is the constant required in $(\mathrm{H} 1)$.

(H3) The functions $F_{i}: \mathbb{R} \times \mathscr{L}^{2}(\mathbf{P} ; \mathbb{4}) \quad \rightarrow$ $\mathscr{L}^{2}(\mathbf{P} ; \mathbb{U}),(t, X) \mapsto F_{i}(t, X)(i=1,2)$, and $G: \mathbb{R} \times \mathscr{L}^{2}(\mathbf{P} ; \mathbb{W}) \rightarrow \mathscr{L}^{2}(\mathbf{P} ; \mathbb{M}),(t, X) \mapsto G(t, X)$ are square-mean almost automorphic in $t \in \mathbb{R}$ for each $X \in \mathscr{L}^{2}(\mathbf{P} ; \mathbb{Z})$. Moreover, $F_{1}, F_{2}$, and $G$ are Lipschitz in $X$ uniformly for $t$ in the following sense: there exist constants $K_{i}>0(i=1,2,3)$ such that

$$
\begin{gathered}
\mathrm{E}\left\|F_{i}(t, X)-F_{i}(t, Y)\right\|^{2} \leq K_{i} \mathrm{E}\|X-Y\|^{2}, \quad i=1,2, \\
\mathbf{E}\|G(t, X)-G(t, Y)\|^{2} \leq K_{3} \mathrm{E}\|X-Y\|^{2}
\end{gathered}
$$

for all stochastic processes $X, Y \in \mathscr{L}^{2}(\mathbf{P} ; \mathbb{U})$ and $t \epsilon$ $\mathbb{R}$.

Definition 9. An $\mathscr{F}_{t}$ progressively measurable process $(X(t))_{t \in \mathbb{R}}$ is called a mild solution of (1) if it satisfies the corresponding stochastic integral equation:

$$
\begin{aligned}
X(t)= & U(t, a) X(a) \\
& +\int_{a}^{t} U(t, \sigma) \int_{a}^{\sigma} C(\sigma-s) G(s, X(s)) d W(s) d \sigma \\
& +\int_{a}^{t} U(t, \sigma) \int_{a}^{\sigma} B(\sigma-s) F_{2}(s, X(s)) d s d \sigma \\
& +\int_{a}^{t} U(t, s) F_{1}(s, X(s)) d s
\end{aligned}
$$

for all $t \geq a$ and each $a \in \mathbb{R}$.

Now we are in a position to show the existence and uniqueness of square-mean almost automorphic solution to (1).

Theorem 10. Assume that conditions (H1)-(H3) are satisfied, then (1) has a unique square-mean almost automorphic mild solution $X(\cdot) \in A A\left(\mathbb{R} ; \mathscr{L}^{2}(\mathbf{P} ; \mathbb{W})\right)$ which can be explicitly expressed as

$$
\begin{aligned}
X(t)= & \int_{-\infty}^{t} U(t, \sigma) \int_{-\infty}^{\sigma} C(\sigma-s) G(s, X(s)) d W(s) d \sigma \\
& +\int_{-\infty}^{t} U(t, \sigma) \int_{-\infty}^{\sigma} B(\sigma-s) F_{2}(s, X(s)) d s d \sigma \\
& +\int_{-\infty}^{t} U(t, s) F_{1}(s, X(s)) d s
\end{aligned}
$$


provided that

$$
\Theta:=3 \frac{M^{2}}{\delta^{2}}\left[K_{1}+K_{2} \cdot\|B\|_{\mathscr{L}^{1}(0, \infty)}^{2}+K_{3} \cdot\|C\|_{\mathscr{L}^{2}(0, \infty)}^{2}\right]<1 .
$$

Proof. First of all, it is not difficult to verify that the stochastic process

$$
\begin{aligned}
X(t)= & \int_{-\infty}^{t} U(t, \sigma) \int_{-\infty}^{\sigma} C(\sigma-s) G(s, X(s)) d W(s) d \sigma \\
& +\int_{-\infty}^{t} U(t, \sigma) \int_{-\infty}^{\sigma} B(\sigma-s) F_{2}(s, X(s)) d s d \sigma \\
& +\int_{-\infty}^{t} U(t, s) F_{1}(s, X(s)) d s
\end{aligned}
$$

is well defined and satisfies

$$
\begin{aligned}
X(t)= & U(t, a) X(a) \\
& +\int_{a}^{t} U(t, \sigma) \int_{a}^{\sigma} C(\sigma-s) G(s, X(s)) d W(s) d \sigma \\
& +\int_{a}^{t} U(t, \sigma) \int_{a}^{\sigma} B(\sigma-s) F_{2}(s, X(s)) d s d \sigma \\
& +\int_{a}^{t} U(t, s) F_{1}(s, X(s)) d s
\end{aligned}
$$

for all $t \geq a$ and each $a \in \mathbb{R}$, and hence it is a mild solution of the original (1).

To seek the square-mean almost automorphic mild solution to (1), let us consider the nonlinear operator $\mathcal{S}$ acting on the Banach space $A A\left(\mathbb{R} ; \mathscr{L}^{2}(\mathbf{P} ; \mathbb{\sharp})\right)$ given by

$$
\begin{aligned}
(\mathcal{S} X)(t):= & \int_{-\infty}^{t} U(t, \sigma) \int_{-\infty}^{\sigma} C(\sigma-s) G(s, X(s)) d W(s) d \sigma \\
& +\int_{-\infty}^{t} U(t, \sigma) \int_{-\infty}^{\sigma} B(\sigma-s) F_{2}(s, X(s)) d s d \sigma \\
& +\int_{-\infty}^{t} U(t, s) F_{1}(s, X(s)) d s .
\end{aligned}
$$

If we can show that the operator $\mathcal{S}$ maps $A A\left(\mathbb{R} ; \mathscr{L}^{2}(\mathbf{P} ; \mathbb{U})\right)$ into itself and it is a contraction mapping, then, by Banach contraction principle, we can conclude that there is a unique square-mean almost automorphic mild solution to (1).

Now define three nonlinear operators acting on the Banach space $A A\left(\mathbb{R} ; \mathscr{L}^{2}(\mathbf{P} ; \mathbb{M})\right)$ as follows:

$$
\begin{gathered}
\left(\mathcal{S}_{1} X\right)(t):=\int_{-\infty}^{t} U(t, s) F_{1}(s, X(s)) d s \\
\left(\mathcal{S}_{2} X\right)(t):=\int_{-\infty}^{t} U(t, \sigma) \int_{-\infty}^{\sigma} B(\sigma-s) F_{2}(s, X(s)) d s d \sigma \\
\left(\mathcal{S}_{3} X\right)(t):=\int_{-\infty}^{t} U(t, \sigma) \int_{-\infty}^{\sigma} C(\sigma-s) G(s, X(s)) d W(s) d \sigma .
\end{gathered}
$$

We show first that $\mathcal{S}_{1} X$ is square-mean almost automorphic whenever $X$ is. Indeed, assuming that $X$ is square-mean almost automorphic, then Lemma 7 implies that $f_{1}(\cdot):=$ $F_{1}(\cdot, X(\cdot))$ is also square-mean almost automorphic. Hence, for any sequence of real numbers $\left\{s_{n}^{\prime}\right\}$, there exists a subsequence $\left\{s_{n}\right\}$ of $\left\{s_{n}^{\prime}\right\}$ and a stochastic process $\widetilde{f_{1}}: \mathbb{R} \rightarrow$ $\mathscr{L}^{2}(\mathbf{P} ; \mathbb{U})$ such that

$$
\begin{aligned}
& \lim _{n \rightarrow \infty} \mathbf{E}\left\|f_{1}\left(t+s_{n}\right)-\widetilde{f}_{1}(t)\right\|^{2}=0, \\
& \lim _{n \rightarrow \infty} \mathbf{E}\left\|\widetilde{f}_{1}\left(t-s_{n}\right)-f_{1}(t)\right\|^{2}=0
\end{aligned}
$$

hold for each $t \in \mathbb{R}$. By assumption (H2), for any $\varepsilon>0$, there exists $N_{1}=N_{1}(\varepsilon) \in \mathbb{N}$ such that $n>N_{1}$ implies that

$$
\begin{gathered}
\left\|U\left(t+s_{n}, s+s_{n}\right)-U(t, s)\right\| \leq \varepsilon e^{-\delta(t-s)} \quad \forall t \geq s, \\
\mathbf{E}\left\|f_{1}\left(t+s_{n}\right)-\widetilde{f}_{1}(t)\right\|^{2}<\varepsilon \\
\mathbf{E}\left\|\widetilde{f}_{1}\left(t-s_{n}\right)-f_{1}(t)\right\|^{2}<\varepsilon \quad \forall t \in \mathbb{R} .
\end{gathered}
$$

Now, define functions on $\mathbb{R}$ as follows:

$$
\begin{aligned}
u(t) & :=\int_{-\infty}^{t} U(t, s) f_{1}(s) d s \\
\widetilde{u}(t) & :=\int_{-\infty}^{t} U(t, s) \widetilde{f_{1}}(s) d s,
\end{aligned}
$$

and then the above observation together with (3.1) and Cauchy-Schwarz inequality implies that for any $\varepsilon>0$ and for the aforementioned $N_{1}=N_{1}(\varepsilon) \in \mathbb{N}$ if $n>N_{1}$ it yields that

$$
\begin{aligned}
& \mathbf{E}\left\|u\left(t+s_{n}\right)-\widetilde{u}(t)\right\|^{2}=\mathbf{E}\left\|\int_{-\infty}^{t+s_{n}} U\left(t+s_{n}, s\right) f_{1}(s) d s-\int_{-\infty}^{t} U(t, s) \widetilde{f}_{1}(s) d s\right\|^{2} \\
&= \mathbf{E} \| \int_{-\infty}^{t} U\left(t+s_{n}, s+s_{n}\right) f_{1}\left(s+s_{n}\right) d s \\
& \quad-\int_{-\infty}^{t} U(t, s) \widetilde{f_{1}}(s) d s \|^{2} \\
& \leq 2 \mathbf{E}\left\|\int_{-\infty}^{t}\left[U\left(t+s_{n}, s+s_{n}\right)-U(t, s)\right] f_{1}\left(s+s_{n}\right) d s\right\|^{2} \\
& \quad+2 \mathbf{E}\left\|\int_{-\infty}^{t} U(t, s)\left[f_{1}\left(s+s_{n}\right)-\widetilde{f}_{1}(s)\right] d s\right\|^{2}
\end{aligned}
$$




$$
\begin{aligned}
& \leq 2 \varepsilon^{2} \mathbf{E}\left(\int_{-\infty}^{t} e^{-\delta(t-s)}\left\|f_{1}\left(s+s_{n}\right)\right\| d s\right)^{2} \\
& +2 M^{2} \mathbf{E}\left(\int_{-\infty}^{t} e^{-\delta(t-s)}\left\|f_{1}\left(s+s_{n}\right)-\widetilde{f_{1}}(s)\right\| d s\right)^{2} \\
& \leq 2 \varepsilon^{2}\left(\int_{-\infty}^{t} e^{-\delta(t-s)} d s\right)\left(\int_{-\infty}^{t} e^{-\delta(t-s)} \mathbf{E}\left\|f_{1}\left(s+s_{n}\right)\right\|^{2} d s\right) \\
& +2 M^{2}\left(\int_{-\infty}^{t} e^{-\delta(t-s)} d s\right) \\
& \times\left(\int_{-\infty}^{t} e^{-\delta(t-s)} \mathbf{E}\left\|f_{1}\left(s+s_{n}\right)-\widetilde{f_{1}}(s)\right\|^{2} d s\right) \\
& \leq 2 \varepsilon^{2}\left(\int_{-\infty}^{t} e^{-\delta(t-s)} d s\right)^{2} \sup _{t \in \mathbb{R}} \mathbf{E}\left\|f_{1}\left(t+s_{n}\right)\right\|^{2} \\
& +2 M^{2}\left(\int_{-\infty}^{t} e^{-\delta(t-s)} d s\right)^{2} \sup _{t \in \mathbb{R}} \mathrm{E}\left\|f_{1}\left(t+s_{n}\right)-\widetilde{f_{1}}(t)\right\|^{2} \\
& \leq \frac{2 M_{1}}{\delta^{2}} \cdot \varepsilon^{2}+\frac{2 M^{2}}{\delta^{2}} \cdot \varepsilon,
\end{aligned}
$$

where $M_{1}:=\sup _{t \in \mathbb{R}} \mathbf{E}\left\|f_{1}(t)\right\|^{2}<+\infty$. Hence,

$$
\lim _{n \rightarrow \infty} \mathbf{E}\left\|u\left(t+s_{n}\right)-\tilde{u}(t)\right\|^{2}=0
$$

for each $t \in \mathbb{R}$. And we can show in a similar way that

$$
\lim _{n \rightarrow \infty} \mathbf{E}\left\|\tilde{u}\left(t-s_{n}\right)-u(t)\right\|^{2}=0
$$

for each $t \in \mathbb{R}$. Thus, we conclude that $u=\mathcal{S}_{1} X \in$ $A A\left(\mathbb{R} ; \mathscr{L}^{2}(\mathbf{P} ; \mathbb{H})\right)$.

In an analogous way, assuming that $X$ is square-mean almost automorphic and using Lemma 7 , one can easily see that $f_{2}(\cdot):=F_{2}(\cdot, X(\cdot))$ is also square-mean almost automorphic. Let $\left\{s_{n}^{\prime}\right\}$ be an arbitrary sequence of real numbers, and then there exists a subsequence $\left\{s_{n}\right\}$ of $\left\{s_{n}^{\prime}\right\}$ and a stochastic process $\widetilde{f_{2}}: \mathbb{R} \rightarrow \mathscr{L}^{2}(\mathbf{P} ; \mathbb{H})$ such that

$$
\begin{aligned}
& \lim _{n \rightarrow \infty} \mathbf{E}\left\|f_{2}\left(t+s_{n}\right)-\widetilde{f_{2}}(t)\right\|^{2}=0, \\
& \lim _{n \rightarrow \infty} \mathbf{E}\left\|\widetilde{f_{2}}\left(t-s_{n}\right)-f_{2}(t)\right\|^{2}=0
\end{aligned}
$$

hold for each $t \in \mathbb{R}$. Now define functions on $\mathbb{R}$ as follows:

$$
\begin{aligned}
& v(t):=\int_{-\infty}^{t} U(t, \sigma) \int_{-\infty}^{\sigma} B(\sigma-s) f_{2}(s) d s d \sigma \\
& \widetilde{v}(t):=\int_{-\infty}^{t} U(t, \sigma) \int_{-\infty}^{\sigma} B(\sigma-s) \widetilde{f_{2}}(s) d s d \sigma
\end{aligned}
$$

and then, by making change of variables $\tau=\sigma-s_{n}$ and $r=$ $s-s_{n}$, we obtain that

$$
\begin{aligned}
& \mathbf{E}\left\|v\left(t+s_{n}\right)-\widetilde{v}(t)\right\|^{2} \\
& =\mathbf{E} \| \int_{-\infty}^{t+s_{n}} U\left(t+s_{n}, \sigma\right) \int_{-\infty}^{\sigma} B(\sigma-s) f_{2}(s) d s d \sigma \\
& -\int_{-\infty}^{t} U(t, \sigma) \int_{-\infty}^{\sigma} B(\sigma-s) \widetilde{f_{2}}(s) d s d \sigma \|^{2} \\
& =\mathbf{E} \| \int_{-\infty}^{t} U\left(t+s_{n}, \sigma+s_{n}\right) \\
& \times \int_{-\infty}^{\sigma} B(\sigma-s) f_{2}\left(s+s_{n}\right) d s d \sigma \\
& -\int_{-\infty}^{t} U(t, \sigma) \int_{-\infty}^{\sigma} B(\sigma-s) \widetilde{f_{2}}(s) d s d \sigma \|^{2} \\
& \leq 2 \mathbf{E} \| \int_{-\infty}^{t}\left[U\left(t+s_{n}, \sigma+s_{n}\right)-U(t, \sigma)\right] \\
& \times \int_{-\infty}^{\sigma} B(\sigma-s) f_{2}\left(s+s_{n}\right) d s d \sigma \|^{2} \\
& +2 \mathbf{E} \| \int_{-\infty}^{t} U(t, \sigma) \int_{-\infty}^{\sigma} B(\sigma-s) \\
& \times\left[f_{2}\left(s+s_{n}\right)-\widetilde{f_{2}}(s)\right] d s d \sigma \|^{2} \\
& \leq 2 \varepsilon^{2} \mathbf{E}\left(\int_{-\infty}^{t} e^{-\delta(t-\sigma)}\right. \\
& \left.\times\left\|\int_{-\infty}^{\sigma} B(\sigma-s) f_{2}\left(s+s_{n}\right) d s\right\| d \sigma\right)^{2} \\
& +2 M^{2} \mathbf{E}\left(\int_{-\infty}^{t} e^{-\delta(t-\sigma)}\right. \\
& \times \| \int_{-\infty}^{\sigma} B(\sigma-s) \\
& \left.\times\left[f_{2}\left(s+s_{n}\right)-\widetilde{f_{2}}(s)\right] d s \| d \sigma\right)^{2} .
\end{aligned}
$$

Let us evaluate the first term of the right-handed side by using Cauchy-Schwarz inequality:

$$
\begin{aligned}
\mathbf{E}\left(\int_{-\infty}^{t} e^{-\delta(t-\sigma)}\left\|\int_{-\infty}^{\sigma} B(\sigma-s) f_{2}\left(s+s_{n}\right) d s\right\| d \sigma\right)^{2} \\
\leq\left(\int_{-\infty}^{t} e^{-\delta(t-\sigma)} d \sigma\right) \\
\quad \times \int_{-\infty}^{t} e^{-\delta(t-\sigma)} \mathbf{E}\left\|\int_{-\infty}^{\sigma} B(\sigma-s) f_{2}\left(s+s_{n}\right) d s\right\|^{2} d \sigma
\end{aligned}
$$


6

Abstract and Applied Analysis

$$
\begin{aligned}
\leq & \left(\int_{-\infty}^{t} e^{-\delta(t-\sigma)} d \sigma\right) \\
& \times\left(\int_{-\infty}^{t} e^{-\delta(t-\sigma)}\left\{\int_{-\infty}^{\sigma}\|B(\sigma-s)\| d s\right\}\right. \\
& \left.\times\left\{\int_{-\infty}^{\sigma}\|B(\sigma-s)\| \mathbf{E}\left\|f_{2}\left(s+s_{n}\right)\right\|^{2} d s\right\} d \sigma\right) \\
\leq & \left\{\int_{-\infty}^{t} e^{-\delta(t-\sigma)} d \sigma\right\}^{2} \cdot\left\{\int_{0}^{+\infty}\|B(u)\| d u\right\}^{2} \\
& \cdot \sup _{s \in \mathbb{R}} \mathbf{E}\left\|f_{2}\left(s+s_{n}\right)\right\|^{2} \leq \frac{\|B\|_{\mathscr{L}^{1}(0, \infty)}^{2}}{\delta^{2}} \cdot \sup _{t \in \mathbb{R}} \mathbf{E}\left\|f_{2}(t)\right\|^{2} .
\end{aligned}
$$

As to the second term, in a similar manner as above, we have the following observation:

$$
\begin{aligned}
& \mathbf{E}\left(\int_{-\infty}^{t} e^{-\delta(t-\sigma)}\left\|\int_{-\infty}^{\sigma} B(\sigma-s)\left[f_{2}\left(s+s_{n}\right)-\widetilde{f_{2}}(s)\right] d s\right\| d \sigma\right)^{2} \\
& \leq \mathbf{E}\left[\left(\int_{-\infty}^{t} e^{-\delta(t-\sigma)} d \sigma\right)\right. \\
& \times\left(\int_{-\infty}^{t} e^{-\delta(t-\sigma)}\right. \\
& \times \| \int_{-\infty}^{\sigma} B(\sigma-s) \\
& \left.\left.\times\left[f_{2}\left(s+s_{n}\right)-\widetilde{f}_{2}(s)\right] d s \|^{2} d \sigma\right)\right] \\
& \leq\left(\int_{-\infty}^{t} e^{-\delta(t-\sigma)} d \sigma\right) \\
& \times\left(\int_{-\infty}^{t} e^{-\delta(t-\sigma)}\right. \\
& \times \mathbf{E} \| \int_{-\infty}^{\sigma} B(\sigma-s) \\
& \left.\times\left[f_{2}\left(s+s_{n}\right)-\widetilde{f_{2}}(s)\right] d s \|^{2} d \sigma\right) \\
& \leq\left(\int_{-\infty}^{t} e^{-\delta(t-\sigma)} d \sigma\right) \\
& \times\left(\int_{-\infty}^{t} e^{-\delta(t-\sigma)}\left\{\int_{-\infty}^{\sigma}\|B(\sigma-s)\| d s\right\}\right. \\
& \times\left\{\int_{-\infty}^{\sigma}\|B(\sigma-s)\|\right. \\
& \left.\left.\times \mathbf{E}\left\|f_{2}\left(s+s_{n}\right)-\widetilde{f_{2}}(s)\right\|^{2} d s\right\} d \sigma\right) \\
& \leq\left\{\int_{-\infty}^{t} e^{-\delta(t-\sigma)} d \sigma\right\}^{2} \cdot\left\{\int_{0}^{+\infty}\|B(u)\| d u\right\}^{2}
\end{aligned}
$$

$$
\begin{aligned}
& \cdot \sup _{t \in \mathbb{R}} \mathbf{E}\left\|f_{2}\left(t+s_{n}\right)-\widetilde{f_{2}}(t)\right\|^{2} \\
\leq & \frac{1}{\delta^{2}} \cdot\|B\|_{\mathscr{L}^{1}(0, \infty)}^{2} \cdot \sup _{t \in \mathbb{R}} \mathbf{E}\left\|f_{2}\left(t+s_{n}\right)-\widetilde{f_{2}}(t)\right\|^{2} \\
\leq & \frac{\|B\|_{\mathscr{L}^{1}(0, \infty)}^{2}}{\delta^{2}} \cdot \varepsilon .
\end{aligned}
$$

Based on the above argument, for arbitrary $\varepsilon>0$, thanks to the boundedness and square-mean almost automorphy of $f_{2}$, there exists certain $N_{2}=N_{2}(\varepsilon) \in \mathbb{N}$ such that $n>N_{2}$ implies that

$$
\begin{aligned}
\mathbf{E}\left\|v\left(t+s_{n}\right)-\widetilde{v}(t)\right\|^{2} & \\
\leq & 2 \varepsilon^{2} \mathbf{E}\left(\int_{-\infty}^{t} e^{-\delta(t-\sigma)}\right. \\
& \left.\times\left\|\int_{-\infty}^{\sigma} B(\sigma-s) f_{2}\left(s+s_{n}\right) d s\right\| d \sigma\right)^{2} \\
& +2 M^{2} \mathbf{E}\left(\int_{-\infty}^{t} e^{-\delta(t-\sigma)}\right. \\
& \times \| \int_{-\infty}^{\sigma} B(\sigma-s) \\
\leq & \left.\frac{2\|B\|_{\mathscr{L}^{1}(0, \infty)}^{2} \cdot \sup _{t \in \mathbb{R}} \mathbf{E}\left\|f_{2}(t)\right\|^{2}}{\delta^{2}} \times\left[f_{2}\left(s+s_{n}\right)-\widetilde{f_{2}}(s)\right] d s \| d \sigma\right)^{2} \\
& \cdot \varepsilon^{2}+\frac{2 M^{2} \cdot\|B\|_{\mathscr{L}^{1}(0, \infty)}^{2} \cdot \varepsilon,}{\delta^{2}}
\end{aligned}
$$

where we use the fact that, for arbitrary $\varepsilon>0$, there exists $N_{2}=N_{2}(\varepsilon) \in \mathbb{N}$ such that, for all $n>N_{2}$,

$$
\mathbf{E}\left\|f_{2}\left(t+s_{n}\right)-\widetilde{f_{2}}(t)\right\|^{2}<\varepsilon
$$

holds for all $t \in \mathbb{R}$. This indicates that

$$
\lim _{n \rightarrow \infty} \mathbf{E}\left\|v\left(t+s_{n}\right)-\widetilde{v}(t)\right\|^{2}=0
$$

for each $t \in \mathbb{R}$. In an analogous way, we can show that

$$
\lim _{n \rightarrow \infty} \mathrm{E}\left\|\widetilde{v}\left(t-s_{n}\right)-v(t)\right\|^{2}=0
$$

for each $t \in \mathbb{R}$. Combining (33) with (34), we obtain that $v=$ $\mathcal{S}_{2} X$ is square-mean almost automorphic.

Assuming that $X \in A A\left(\mathbb{R} ; \mathscr{L}^{2}(\mathbf{P} ; \mathbb{H})\right)$, then similar argument as above ensures that $g(\cdot):=G(\cdot, X(\cdot)) \in$ $A A\left(\mathbb{R} ; \mathscr{L}^{2}(\mathbf{P} ; \mathbb{M})\right)$. As a consequence, for every sequence of real numbers $\left\{s_{n}^{\prime}\right\}$ there exist a subsequence $\left\{s_{n}\right\} \subset\left\{s_{n}^{\prime}\right\}$ and a stochastic process $\tilde{g}$ such that

$$
\begin{aligned}
& \lim _{n \rightarrow \infty} \mathbf{E}\left\|g\left(t+s_{n}\right)-\tilde{g}(t)\right\|^{2}=0, \\
& \lim _{n \rightarrow \infty} \mathbf{E}\left\|\tilde{g}\left(t-s_{n}\right)-g(t)\right\|^{2}=0
\end{aligned}
$$


Abstract and Applied Analysis

7

hold for each $t \in \mathbb{R}$. Hence, for arbitrary $\varepsilon>0$, there exists certain $N_{3}=N_{3}(\varepsilon) \in \mathbb{N}$ such that, for all $n>N_{3}$, there holds

$$
\mathbf{E}\left\|g\left(t+s_{n}\right)-\tilde{g}(t)\right\|^{2}<\varepsilon
$$

for all $t \in \mathbb{R}$.

The next step aims to prove the square-mean almost automorphy of $\mathcal{S}_{3} X$. This is more complicated because the involvement of the Brownian motion $W$. Consider the Brownian motion $\widetilde{W}$ defined by

$$
\widetilde{W}(s)=W\left(s+s_{n}\right)-W\left(s_{n}\right)
$$

for each $s \in \mathbb{R}$, which has the same distribution as $W$. Define two functions on $\mathbb{R}$ as below:

$$
\begin{aligned}
& \omega(t):=\int_{-\infty}^{t} U(t, \sigma) \int_{-\infty}^{\sigma} C(\sigma-s) g(s) d W(s) d \sigma, \\
& \widetilde{\omega}(t):=\int_{-\infty}^{t} U(t, \sigma) \int_{-\infty}^{\sigma} C(\sigma-s) \tilde{g}(s) d W(s) d \sigma .
\end{aligned}
$$

By making change of variables $\tau=\sigma-s_{n}$ and $r=s-s_{n}$, and then using Cauchy-Schwarz inequality, we obtain that

$$
\begin{aligned}
& \mathbf{E}\left\|\omega\left(t+s_{n}\right)-\widetilde{\omega}(t)\right\|^{2} \\
& =\mathbf{E} \| \int_{-\infty}^{t+s_{n}} U\left(t+s_{n}, \sigma\right) \int_{-\infty}^{\sigma} C(\sigma-s) g(s) d W(s) d \sigma \\
& -\int_{-\infty}^{t} U(t, \sigma) \int_{-\infty}^{\sigma} C(\sigma-s) \tilde{g}(s) d W(s) d \sigma \|^{2} \\
& =\mathbf{E} \| \int_{-\infty}^{t} U\left(t+s_{n}, \sigma+s_{n}\right) \\
& \times \int_{-\infty}^{\sigma} C(\sigma-s) g\left(s+s_{n}\right) d W\left(s+s_{n}\right) d \sigma \\
& -\int_{-\infty}^{t} U(t, \sigma) \int_{-\infty}^{\sigma} C(\sigma-s) \tilde{g}(s) d W(s) d \sigma \|^{2} \\
& =\mathbf{E} \| \int_{-\infty}^{t} U\left(t+s_{n}, \sigma+s_{n}\right) \\
& \times \int_{-\infty}^{\sigma} C(\sigma-s) g\left(s+s_{n}\right) d \widetilde{W}(s) d \sigma \\
& -\int_{-\infty}^{t} U(t, \sigma) \int_{-\infty}^{\sigma} C(\sigma-s) \tilde{g}(s) d \widetilde{W}(s) d \sigma \|^{2} \\
& \leq 2 \mathbf{E} \| \int_{-\infty}^{t}\left[U\left(t+s_{n}, \sigma+s_{n}\right)-U(t, \sigma)\right] \\
& \times \int_{-\infty}^{\sigma} C(\sigma-s) g\left(s+s_{n}\right) d \widetilde{W}(s) d \sigma \|^{2}
\end{aligned}
$$

$$
\begin{aligned}
& +2 \mathbf{E} \| \int_{-\infty}^{t} U(t, \sigma) \\
& \times \int_{-\infty}^{\sigma} C(\sigma-s) \\
& \times\left[g\left(s+s_{n}\right)-\widetilde{g}(s)\right] d \widetilde{W}(s) d \sigma \|^{2} \\
& \leq 2 \varepsilon^{2} \mathbf{E}\left(\int_{-\infty}^{t} e^{-\delta(t-\sigma)}\right. \\
& \left.\times\left\|\int_{-\infty}^{\sigma} C(\sigma-s) g\left(s+s_{n}\right) d \widetilde{W}(s)\right\| d \sigma\right)^{2} \\
& +2 M^{2} \mathbf{E}\left(\int_{-\infty}^{t} e^{-\delta(t-\sigma)}\right. \\
& \times \| \int_{-\infty}^{\sigma} C(\sigma-s) \\
& \times\left[g\left(s+s_{n}\right)\right. \\
& -\widetilde{g}(s)] d \widetilde{W}(s) \| d \sigma)^{2} \\
& \leq 2 \varepsilon^{2}\left(\int_{-\infty}^{t} e^{-\delta(t-\sigma)} d \sigma\right) \\
& \times\left(\int_{-\infty}^{t} e^{-\delta(t-\sigma)}\right. \\
& \left.\times \mathbf{E}\left\|\int_{-\infty}^{\sigma} C(\sigma-s) g\left(s+s_{n}\right) d \widetilde{W}(s)\right\|^{2} d \sigma\right) \\
& +2 M^{2}\left(\int_{-\infty}^{t} e^{-\delta(t-\sigma)} d \sigma\right) \\
& \times\left(\int_{-\infty}^{t} e^{-\delta(t-\sigma)}\right. \\
& \times \mathbf{E} \| \int_{-\infty}^{\sigma} C(\sigma-s) \\
& \left.\times\left[g\left(s+s_{n}\right)-\widetilde{g}(s)\right] d \widetilde{W}(s) \|^{2} d \sigma\right) .
\end{aligned}
$$

For the above-mentioned $\varepsilon>0$, by using an estimate on Ito integral established in [26], it follows that once $n>N_{3}$, then the first term of the above inequality can be evaluated as

$$
\begin{aligned}
& \left(\int_{-\infty}^{t} e^{-\delta(t-\sigma)} d \sigma\right) \\
& \quad \times\left(\int_{-\infty}^{t} e^{-\delta(t-\sigma)}\right. \\
& \left.\quad \times \mathbf{E}\left\|\int_{-\infty}^{\sigma} C(\sigma-s) g\left(s+s_{n}\right) d \widetilde{W}(s)\right\|^{2} d \sigma\right)
\end{aligned}
$$


8

Abstract and Applied Analysis

$$
\begin{aligned}
\leq & \left(\int_{-\infty}^{t} e^{-\delta(t-\sigma)} d \sigma\right) \\
& \times\left(\int_{-\infty}^{t} e^{-\delta(t-\sigma)}\right. \\
& \left.\times\left\{\int_{-\infty}^{\sigma} \mathbf{E}\left\|C(\sigma-s) g\left(s+s_{n}\right)\right\|^{2} d s\right\} d \sigma\right) \\
\leq & \left(\int_{-\infty}^{t} e^{-\delta(t-\sigma)} d \sigma\right) \\
& \times\left(\int_{-\infty}^{t} e^{-\delta(t-\sigma)}\right. \\
\leq & \left(\int_{-\infty}^{t} e^{-\delta(t-\sigma)} d \sigma\right) \\
& \left.\times \|_{\mathscr{L}^{2}(0, \infty)}^{\delta^{2}} \cdot \int_{-\infty} e^{-\delta(t-\sigma)} d \sigma\right)^{2} \cdot\left(\int_{0}^{\infty}\|C(u)\|^{2} d u\right) \\
& \times\left(\int_{-\infty}^{t} e^{-\delta(t-\sigma)}\left\{\int_{-\infty}^{\sigma}\|C(\sigma-s)\|^{2} d s\right\} d \sigma\right) \\
& \left.\left.\cdot \sup _{s \in \mathbb{R}} \mathbf{E}\left\|g\left(s+s_{n}\right)\right\|^{2} \mathbf{E}\left\|g\left(s+s_{n}\right)\right\|^{2} d s\right\} d \sigma\right) \\
& \left(\int_{t}^{t} \|^{2},\right.
\end{aligned}
$$

and the second term can be evaluated analogously as

$$
\begin{aligned}
& \left(\int_{-\infty}^{t} e^{-\delta(t-\sigma)} d \sigma\right) \\
& \quad \times\left(\int_{-\infty}^{t} e^{-\delta(t-\sigma)} \mathbf{E} \| \int_{-\infty}^{\sigma} C(\sigma-s)\right. \\
& \left.\times\left[g\left(s+s_{n}\right)-\widetilde{g}(s)\right] d \widetilde{W}(s) \|^{2} d \sigma\right) \\
& \leq\left(\int_{-\infty}^{t} e^{-\delta(t-\sigma)} d \sigma\right) \\
& \quad \times\left(\int_{-\infty}^{t} e^{-\delta(t-\sigma)}\left\{\int_{-\infty}^{\sigma} \mathbf{E}\left\|C(\sigma-s)\left[g\left(s+s_{n}\right)-\widetilde{g}(s)\right]\right\|^{2} d s\right\} d \sigma\right) \\
& \leq\left(\int_{-\infty}^{t} e^{-\delta(t-\sigma)} d \sigma\right) \\
& \quad \times\left(\int _ { - \infty } ^ { t } e ^ { - \delta ( t - \sigma ) } \left\{\int_{-\infty}^{\sigma}\|C(\sigma-s)\|^{2} \mathbf{E}\right.\right. \\
& \left.\left.\times\left\|g\left(s+s_{n}\right)-\widetilde{g}(s)\right\|^{2} d s\right\} d \sigma\right)
\end{aligned}
$$

$$
\begin{aligned}
& \leq\left(\int_{-\infty}^{t} e^{-\delta(t-\sigma)} d \sigma\right)\left(\int_{-\infty}^{t} e^{-\delta(t-\sigma)}\right. \\
& \left.\quad \times\left\{\int_{-\infty}^{\sigma}\|C(\sigma-s)\|^{2} d s\right\} d \sigma\right) \\
& \quad \cdot \sup _{t \in \mathbb{R}}\left\|g\left(s+s_{n}\right)-\tilde{g}(s)\right\|^{2} \\
& \leq\left(\int_{-\infty}^{t} e^{-\delta(t-\sigma)} d \sigma\right)^{2} \\
& \quad \cdot\left(\int_{0}^{\infty}\|C(u)\|^{2} d u\right) \cdot \sup _{t \in \mathbb{R}}\left\|g\left(s+s_{n}\right)-\tilde{g}(s)\right\|^{2} \\
& \leq \frac{\|C\|_{\mathscr{L}^{2}(0, \infty)}^{2} \cdot \varepsilon .}{\delta^{2}}
\end{aligned}
$$

The above argument yields that

$$
\begin{aligned}
& \mathbf{E}\left\|\omega\left(t+s_{n}\right)-\widetilde{\omega}(t)\right\|^{2} \\
& \leq 2 \varepsilon^{2}\left(\int_{-\infty}^{t} e^{-\delta(t-\sigma)} d \sigma\right) \\
& \times\left(\int_{-\infty}^{t} e^{-\delta(t-\sigma)}\right. \\
& \left.\times \mathbf{E}\left\|\int_{-\infty}^{\sigma} C(\sigma-s) g\left(s+s_{n}\right) d \widetilde{W}(s)\right\|^{2} d \sigma\right) \\
& +2 M^{2}\left(\int_{-\infty}^{t} e^{-\delta(t-\sigma)} d \sigma\right) \\
& \times\left(\int_{-\infty}^{t} e^{-\delta(t-\sigma)}\right. \\
& \times \mathbf{E} \| \int_{-\infty}^{\sigma} C(\sigma-s) \\
& \left.\times\left[g\left(s+s_{n}\right)-\widetilde{g}(s)\right] d \widetilde{W}(s) \|^{2} d \sigma\right) \\
& \leq \frac{2\|C\|_{\mathscr{L}^{2}(0, \infty)}^{2} \cdot \sup _{t \in \mathbb{R}} \mathbf{E}\|g(t)\|^{2}}{\delta^{2}} \cdot \varepsilon^{2} \\
& +\frac{2 M^{2} \cdot\|C\|_{\mathscr{L}^{2}(0, \infty)}^{2}}{\delta^{2}} \cdot \mathcal{\varepsilon}
\end{aligned}
$$

which implies that

$$
\lim _{n \rightarrow \infty} \mathbf{E}\left\|\omega\left(t+s_{n}\right)-\widetilde{\omega}(t)\right\|^{2}=0
$$

for each $t \in \mathbb{R}$. Analogously, we can show that

$$
\lim _{n \rightarrow \infty} \mathbf{E}\left\|\widetilde{\omega}\left(t-s_{n}\right)-\omega(t)\right\|^{2}=0
$$

for each $t \in \mathbb{R}$. Combining (43) with (44), we obtain that $\omega=\mathcal{S}_{3} X$ is square-mean almost automorphic. 
In view of the above arguments, it follows from (18) that the nonlinear operator $\delta=\delta_{1}+\delta_{2}+\delta_{3}$ maps $A A\left(\mathbb{R} ; \mathscr{L}^{2}(\mathbf{P} ; \mathbb{U})\right)$ into itself. To complete the proof, it suffices to show that $\mathcal{S}$ is a contraction mapping on $A A\left(\mathbb{R} ; \mathscr{L}^{2}(\mathbf{P} ; \mathbb{W})\right)$. Indeed, for each $X, Y \in A A\left(\mathbb{R} ; \mathscr{L}^{2}(\mathbf{P} ; \mathbb{W})\right)$, thanks to the fact that $(a+b+c)^{2} \leq 3 a^{2}+3 b^{2}+3 c^{2}$, we have the following observation:

$$
\begin{aligned}
& \mathbf{E}\|(\mathcal{S} X)(t)-(\mathcal{S} Y)(t)\|^{2} \\
& \leq 3 \mathrm{E}\left\|\int_{-\infty}^{t} U(t, s)\left[F_{1}(s, X(s))-F_{1}(s, Y(s))\right] d s\right\|^{2} \\
& +3 \mathrm{E} \| \int_{-\infty}^{t} U(t, \sigma) \\
& \times \int_{-\infty}^{\sigma} B(\sigma-s) \\
& \times\left[F_{2}(s, X(s))-F_{2}(s, Y(s))\right] d s d \sigma \|^{2} \\
& +3 \mathrm{E} \| \int_{-\infty}^{t} U(t, \sigma) \\
& \times \int_{-\infty}^{\sigma} C(\sigma-s) \\
& \times[G(s, X(s))-G(s, Y(s))] d W(s) d \sigma \|^{2} \\
& \leq 3 \mathrm{E}\left(\int_{-\infty}^{t}\|U(t, s)\|\left\|F_{1}(s, X(s))-F_{1}(s, Y(s))\right\| d s\right)^{2} \\
& +3 \mathbf{E}\left(\int_{-\infty}^{t}\|U(t, \sigma)\|\right. \\
& \times \int_{-\infty}^{\sigma} \| B(\sigma-s) \\
& \left.\times\left[F_{2}(s, X(s))-F_{2}(s, Y(s))\right] \| d s d \sigma\right)^{2} \\
& +3 \mathrm{E}\left(\int_{-\infty}^{t}\|U(t, \sigma)\|\right. \\
& \times \| \int_{-\infty}^{\sigma} C(\sigma-s)[G(s, X(s)) \\
& -G(s, Y(s))] d W(s) \| d \sigma)^{2} .
\end{aligned}
$$

Now, we evaluate the first term of the right-hand side with the help of Cauchy-Schwarz inequality as follows:

$$
\begin{aligned}
3 \mathrm{E}\left(\int_{-\infty}^{t}\|U(t, s)\|\left\|F_{1}(s, X(s))-F_{1}(s, Y(s))\right\| d s\right)^{2} \\
\quad \leq 3 M^{2} \mathrm{E}\left(\int_{-\infty}^{t} e^{-\delta(t-s)}\left\|F_{1}(s, X(s))-F_{1}(s, Y(s))\right\| d s\right)^{2}
\end{aligned}
$$

$$
\begin{aligned}
\leq & 3 M^{2}\left(\int_{-\infty}^{t} e^{-\delta(t-s)} d s\right) \\
& \times\left(\int_{-\infty}^{t} e^{-\delta(t-s)} \mathbf{E}\left\|F_{1}(s, X(s))-F_{1}(s, Y(s))\right\|^{2} d s\right) \\
\leq & 3 M^{2}\left(\int_{-\infty}^{t} e^{-\delta(t-s)} d s\right)^{2} \\
& \times \sup _{s \in \mathbb{R}} \mathbf{E} F_{1}(s, X(s))-F_{1}(s, Y(s)) \|^{2} \\
\leq & 3 K_{1} M^{2}\left(\int_{-\infty}^{t} e^{-\delta(t-s)} d s\right)^{2} \sup _{t \in \mathbb{R}} \mathbf{E}\|X(t)-Y(t)\|^{2} \\
= & \frac{3 K_{1}}{\delta^{2}} \cdot M^{2} \cdot \sup _{t \in \mathbb{R}} \mathbf{E}\|X(t)-Y(t)\|^{2} .
\end{aligned}
$$

As to the second term, we can proceed in the same manner as above and obtain

$$
\begin{aligned}
& 3 \mathrm{E}\left(\int_{-\infty}^{t}\|U(t, \sigma)\|\right. \\
& \left.\quad \times \int_{-\infty}^{\sigma}\left\|B(\sigma-s)\left[F_{2}(s, X(s))-F_{2}(s, Y(s))\right]\right\| d s d \sigma\right)^{2} \\
& \quad \leq \frac{3 K_{2}}{\delta^{2}} \cdot M^{2} \cdot\|B\|_{\mathscr{L}^{1}(0, \infty)}^{2} \cdot \sup _{t \in \mathbb{R}} \mathrm{E}\|X(t)-Y(t)\|^{2} .
\end{aligned}
$$

As far as the last term of the right-hand side is concerned, we use again the estimate on the Ito integral to obtain

$$
\begin{array}{r}
3 \mathbf{E}\left(\int_{-\infty}^{t}\|U(t, \sigma)\|\right. \\
\times \| \int_{-\infty}^{\sigma} C(\sigma-s) \\
\quad \times[G(s, X(s))-G(s, Y(s))] d W(s) \| d \sigma)^{2} \\
\leq 3 M^{2} \mathbf{E}\left(\int_{-\infty}^{t} e^{-\delta(t-\sigma)}\right. \\
\quad \times \| \int_{-\infty}^{\sigma} C(\sigma-s) \\
\times[G(s, X(s))-G(s, Y(s))] d W \| d \sigma)^{2}
\end{array}
$$




$$
\begin{aligned}
& \leq 3 M^{2}\left(\int_{-\infty}^{t} e^{-\delta(t-\sigma)} d \sigma\right) \\
& \times\left(\int_{-\infty}^{t} e^{-\delta(t-\sigma)}\right. \\
& \times \mathbf{E} \| \int_{-\infty}^{\sigma} C(\sigma-s) \\
& \left.\times[G(s, X(s))-G(s, Y(s))] d W(s) \|^{2} d \sigma\right) \\
& \leq 3 M^{2}\left(\int_{-\infty}^{t} e^{-\delta(t-\sigma)} d \sigma\right) \\
& \times\left(\int_{-\infty}^{t} e^{-\delta(t-\sigma)}\right. \\
& \times \int_{-\infty}^{\sigma}\|C(\sigma-s)\|^{2} \\
& \left.\times \mathbf{E}\|G(s, X(s))-G(s, Y(s))\|^{2} d s d \sigma\right) \\
& \leq 3 M^{2}\left(\int_{-\infty}^{t} e^{-\delta(t-\sigma)} d \sigma\right)^{2} \cdot\|C\|_{\mathscr{L}^{2}(0, \infty)}^{2} \\
& \text { · } \sup _{s \in \mathbb{R}} \mathbf{E}\|G(s, X(s))-G(s, Y(s))\|^{2} \\
& \leq 3 K_{3} M^{2}\left(\int_{-\infty}^{t} e^{-\delta(t-\sigma)} d \sigma\right)^{2} \cdot\|C\|_{\mathscr{L}^{2}(0, \infty)}^{2} \\
& \cdot \sup _{t \in \mathbb{R}} \mathbf{E}\|X(t)-Y(t)\|^{2} \\
& =\frac{3 K_{3}}{\delta^{2}} \cdot M^{2} \cdot\|C\|_{\mathscr{L}^{2}(0, \infty)}^{2} \cdot \sup _{t \in \mathbb{R}} \mathrm{E}\|X(t)-Y(t)\|^{2} .
\end{aligned}
$$

Thus, by combining the three inequalities together, we obtain that, for each $t \in \mathbb{R}$,

$$
\begin{aligned}
& \mathrm{E}\|(\mathcal{S} X)(t)-(\mathcal{S} Y)(t)\|^{2} \\
& \leq\left\{3 \frac{M^{2}}{\delta^{2}}\left[K_{1}+K_{2} \cdot\|B\|_{\mathscr{L}^{1}(0, \infty)}^{2}+K_{3} \cdot\|C\|_{\mathscr{L}^{2}(0, \infty)}^{2}\right]\right\} \\
& \cdot \sup _{t \in \mathbb{R}} \mathrm{E}\|X(t)-Y(t)\|^{2} .
\end{aligned}
$$

That is,

$$
\|(\mathcal{S} X)(t)-(\mathcal{S} Y)(t)\|_{2}^{2} \leq \Theta \sup _{t \in \mathbb{R}}\|X(t)-Y(t)\|_{2}^{2},
$$

where $\Theta:=3\left(M^{2} / \delta^{2}\right)\left[K_{1}+K_{2} \cdot\|B\|_{\mathscr{L}^{1}(0, \infty)}^{2}+K_{3} \cdot\|C\|_{\mathscr{L}^{2}(0, \infty)}^{2}\right]$. Notice that

$$
\sup _{t \in \mathbb{R}}\|X(t)-Y(t)\|_{2}^{2} \leq\left(\sup _{t \in \mathbb{R}}\|X(t)-Y(t)\|_{2}\right)^{2} .
$$

As a result, (50) together with (51) gives that, for each $t \in \mathbb{R}$,

$$
\begin{aligned}
& \|(\mathcal{S} X)(t)-(\mathcal{S} Y)(t)\|_{2} \leq \sqrt{\Theta} \sup _{t \in \mathbb{R}}\|X(t)-Y(t)\|_{2} \\
& =\sqrt{\Theta}\|X-Y\|_{\infty} .
\end{aligned}
$$

It follows that

$$
\begin{aligned}
\| \mathcal{S} X & -\mathcal{S} Y \|_{\infty} \\
& =\sup _{t \in \mathbb{R}}\|(\mathcal{S} X)(t)-(\mathcal{S} Y)(t)\|_{2} \leq \sqrt{\Theta}\|X-Y\|_{\infty},
\end{aligned}
$$

which implies that $\mathcal{S}$ is a contraction mapping by the assumption (15) imposed on $\Theta$. Therefore, by the Banach contraction principle, we conclude that there exists a unique fixed point $X$ for $\mathcal{S}$ in $A A\left(\mathbb{R} ; \mathscr{L}^{2}(\mathbf{P} ; \mathbb{H})\right)$, which is the unique squaremean almost automorphic mild solution to the functional integrodifferential semilinear stochastic evolution equation (1) as we have claimed. The proof is complete.

Remark 11. If the functions $F_{1}, F_{2}, G$ in (1) are square-mean almost periodic in $t$, then the unique square-mean almost automorphic solution obtained in Theorem 10 is actually square-mean almost periodic; see paper [27].

Now we are in a position to show the asymptotically stable property of the unique square-mean almost automorphic solution to (1). Recall that the unique square-mean almost automorphic solution $X^{*}(t)$ of (1) is said to be stable in square-mean sense if, for arbitrary $\epsilon>0$, there exists $\delta>0$ such that

$$
\mathbf{E}\left\|X(t)-X^{*}(t)\right\|^{2}<\epsilon, \quad t \geq 0
$$

whenever $\mathbf{E}\left\|X(0)-X^{*}(0)\right\|^{2}<\delta$, where $X(t)$ stands for a solution of (1) with initial value $X(0)$. The solution $X^{*}(t)$ is said to be asymptotically stable in square-mean sense if it is stable in square-mean sense and

$$
\lim _{t \rightarrow \infty} \mathbf{E}\left\|X(t)-X^{*}(t)\right\|^{2}=0
$$

The following Gronwall-type inequality is proved to be useful in our asymptotic stability analysis.

Lemma 12. Let $u(t), b(t)$ be nonnegative continuous functions for $t \geq a$, and $\alpha, \gamma$ be some positive constants. If

$$
u(t) \leq \alpha e^{-\beta(t-a)}+\int_{a}^{t} e^{-\beta(t-s)} b(s) u(s) d s, \quad t \geq a,
$$

then

$$
u(t) \leq \alpha \exp \left\{-\beta(t-a)+\int_{a}^{t} b(s) d s\right\} .
$$

Theorem 13. Let all the assumptions in Theorem 10 hold and assume that

$$
\frac{M^{2}}{\delta^{2}}\left[K_{1}+K_{2} \cdot\|B\|_{\mathscr{L}^{1}(0, \infty)}^{2}+K_{3} \cdot\|C\|_{\mathscr{L}^{2}(0, \infty)}^{2}\right]<\frac{1}{4} .
$$

Then the unique square-mean almost automorphic mild solution $X^{*}(t)$ of $(1)$ is asymptotically stable in square-mean sense. 
Proof. Let $X(t)$ be any mild solution of (1) with initial value $X(0)$. Then, on account of $(\mathrm{H} 1)-(\mathrm{H} 2)$ and the assumptions imposed on $B$ and $C$, along the same line as in [9] we could show that, for any $t \geq 0$,

$$
\begin{aligned}
& \mathbf{E}\left\|X(t)-X^{*}(t)\right\|^{2} \\
& =\mathbf{E} \| U(t, 0)\left[X(0)-X^{*}(0)\right] \\
& +\int_{0}^{t} U(t, s) \\
& \times\left[F_{1}(s, X(s))-F_{1}\left(s, X^{*}(s)\right)\right] d s \\
& +\int_{0}^{t} U(t, \sigma) \\
& \times \int_{0}^{\sigma} B(\sigma-s) \\
& \times\left[F_{2}(s, X(s))-F_{2}\left(s, X^{*}(s)\right)\right] d s d \sigma \\
& +\int_{0}^{t} U(t, \sigma) \\
& \times \int_{0}^{\sigma} C(\sigma-s) \\
& \times\left[G(s, X(s))-G\left(s, X^{*}(s)\right)\right] d W(s) d \sigma \|^{2} \\
& \leq 4 \mathbf{E}\left\|U(t, 0)\left[X(0)-X^{*}(0)\right]\right\|^{2} \\
& +4 \mathbf{E}\left\|\int_{0}^{t} U(t, s)\left[F_{1}(s, X(s))-F_{1}\left(s, X^{*}(s)\right)\right]\right\|^{2} \\
& +4 \mathbf{E} \| \int_{0}^{t} U(t, \sigma) \\
& \times \int_{0}^{\sigma} B(\sigma-s) \\
& \times\left[F_{2}(s, X(s))-F_{2}\left(s, X^{*}(s)\right)\right] d s d \sigma \|^{2} \\
& +4 \mathbf{E} \| \int_{0}^{t} U(t, \sigma) \\
& \times \int_{0}^{\sigma} C(\sigma-s) \\
& \times\left[G(s, X(s))-G\left(s, X^{*}(s)\right)\right] d W(s) d \sigma \|^{2} \\
& \leq 4 M^{2} e^{-\delta t} \mathbf{E}\left\|X(0)-X^{*}(0)\right\|^{2} \\
& +\kappa \int_{0}^{t} e^{-\delta(t-s)} \mathbf{E}\left\|X(s)-X^{*}(s)\right\|^{2} d s,
\end{aligned}
$$

where $\kappa:=\left(4 M^{2}\left(K_{1}+K_{2} \cdot\|B\|_{\mathscr{L}^{1}(0, \infty)}^{2}+K_{3} \cdot\|C\|_{\mathscr{L}^{2}(0, \infty)}^{2}\right)\right) / \delta$.
Define $Y(t):=\mathbf{E}\left\|X(t)-X^{*}(t)\right\|^{2}$, and it yields that

$$
Y(t) \leq 4 M^{2} Y(0) e^{-\delta t}+\kappa \int_{0}^{t} e^{-\delta(t-s)} Y(s) d s .
$$

Hence, it follows from Lemma 12 that

$$
Y(t) \leq 4 M^{2} Y(0) \exp \{(-\delta+\kappa) t\}
$$

Straightforwardly, we obtain that $Y(t)$ converges to 0 exponentially fast if $-\delta+\kappa<0$, which is equivalent to our condition (58). Thus, we come to the conclusion that the unique square-mean almost automorphic mild solution $X^{*}(t)$ of (1) is asymptotically stable in square-mean sense. The proof is completed.

\section{Applications}

To illustrate the applications of our abstract results, let us consider the following nonautonomous functional integrodifferential stochastic partial differential equation:

$$
\begin{aligned}
\frac{\partial X}{\partial t}= & \frac{\partial^{2} X}{\partial x^{2}}+\int_{-\infty}^{t} C(t-s) G(s, X(s, x)) d W(s) \\
& +\int_{-\infty}^{t} B(t-s) F_{2}(s, X(s, x)) d s+F_{1}(t, X(t, x))
\end{aligned}
$$

for $t \in \mathbb{R}$ and $x \in \Omega$, where $\Omega \subset \mathbb{R}^{n}$ is a bounded subset whose boundary $\partial \Omega$ is both of class $C^{2}$ and locally on one side of $\Omega$. Suppose further that (62) satisfies the following boundary conditions:

$$
\sum_{i, j=1}^{n} n_{i}(x) a_{i j}(t, x) \frac{d X(t, x)}{d x_{i}}=0, \quad t \in \mathbb{R}, x \in \partial \Omega,
$$

where $n(x)=\left(n_{1}(x), n_{2}(x), \ldots, n_{n}(x)\right)$ is the outer unit normal vector. A family of operators $A(t, x)$ defined by $\partial^{2} X / \partial x^{2}=A(t, x) X(t, x)$ is formally assigned to be

$$
A(t, x)=\sum_{i, j=1}^{n} \frac{\partial}{\partial x_{i}}\left(a_{i j}(t, x) \frac{\partial}{\partial x_{j}}\right)+c(t, x), \quad t \in \mathbb{R}, x \in \Omega,
$$

where $a_{i j}(i, j=1,2, \ldots, n)$ and $c$ satisfy the following conditions.

(H4) The coefficients $a_{i j}(i, j=1,2, \ldots, n)$ are symmetric, that is, $a_{i j}=a_{j i}$ for all $i, j=1,2, \ldots, n$. In addition,

$$
\begin{aligned}
a_{i j} \in & C_{b}^{\mu}\left(\mathbb{R} ; \mathscr{L}^{2}(\mathbf{P} ; C(\bar{\Omega}))\right) \\
& \cap C_{b}\left(\mathbb{R} ; \mathscr{L}^{2}\left(\mathbf{P} ; C^{1}(\bar{\Omega})\right)\right) \\
& \cap A A\left(\mathbb{R} ; \mathscr{L}^{2}\left(\mathbf{P} ; \mathscr{L}^{2}(\Omega)\right)\right)
\end{aligned}
$$

for all $i, j=1,2, \ldots, n$ and

$$
\begin{aligned}
c \in C_{b}^{\mu}\left(\mathbb{R} ; \mathscr{L}^{2}\left(\mathbf{P} ; \mathscr{L}^{2}(\Omega)\right)\right) \cap C_{b}\left(\mathbb{R} ; \mathscr{L}^{2}(\mathbf{P} ; C(\bar{\Omega}))\right) \\
\cap A A\left(\mathbb{R} ; \mathscr{L}^{2}\left(\mathbf{P} ; \mathscr{L}^{1}(\Omega)\right)\right)
\end{aligned}
$$


for some $\mu \in(1 / 2,1]$, where $\bar{\Omega}$ means the closure of $\Omega$.

(H5) There exists $\delta_{0}>0$ such that

$$
\sum_{i, j=1}^{n} a_{i j}(t, x) \eta_{i} \eta_{j} \geq \delta_{0}|\eta|^{2}
$$

for all $(t, x) \in \mathbb{R} \times \bar{\Omega}$ and $\eta \in \mathbb{R}^{n}$.

Now, let $\mathbb{H}=\mathscr{L}^{2}(\Omega)$ and let $\mathscr{H}^{2}(\Omega)$ be the Sobolev space of order 2 on $\Omega$. For each $t \in \mathbb{R}$, define an operator $A(t)$ on $\mathscr{L}^{2}(\mathbf{P} ; \mathbb{H})$ by

$$
A(t) X=A(t, x) X \quad \forall X \in \mathscr{D}(A(t)),
$$

where

$$
\begin{aligned}
\mathscr{D}(A(t))= & \left\{X \in \mathscr{L}^{2}\left(\mathbf{P}, \mathscr{H}^{2}(\Omega)\right):\right. \\
& \left.\sum_{i, j=1}^{n} n_{i}(\cdot) a_{i j}(t, \cdot) \frac{d X(t, \cdot)}{d x_{i}}=0 \text { on } \partial \Omega\right\} .
\end{aligned}
$$

Under assumptions (H4)-(H5), the existence of an evolution family $U(t, s)$ satisfying (H1) is guaranteed; see, for example, [28].

And thus, as an immediate consequence of Theorem 10, it yields the following.

Theorem 14. Under assumptions (H2), (H3), (H4), and (H5), the nonautonomous integrodifferential stochastic evolution equation (62)-(63) has a unique mild solution which is squaremean almost automorphic provided that (15) holds. If, in addition, (58) is valid, then the unique almost automorphic solution is asymptotically stable in square-mean sense.

\section{Acknowledgments}

This paper is jointly supported by the National Natural Science Foundation of China under Grant nos. 11201266 and 11171191 and the Tianyuan Youth Foundation of National Natural Science Foundation of China under Grant nos. 11026150 and 11026098.

\section{References}

[1] P. H. Bezandry, "Existence of almost periodic solutions to some functional integro-differential stochastic evolution equations," Statistics \& Probability Letters, vol. 78, no. 17, pp. 2844-2849, 2008.

[2] P. H. Bezandry and T. Diagana, "Existence of almost periodic solutions to some stochastic differential equations," Applicable Analysis, vol. 86, no. 7, pp. 819-827, 2007.

[3] P. H. Bezandry and T. Diagana, "Square-mean almost periodic solutions nonautonomous stochastic differential equations," Electronic Journal of Differential Equations, vol. 2007, no. 117, 10 pages, 2007.
[4] P. H. Bezandry and T. Diagana, "Existence of $S^{2}$-almost periodic solutions to a class of nonautonomous stochastic evolution equations," Electronic Journal of Qualitative Theory of Differential Equations, vol. 35, pp. 1-19, 2008.

[5] A. Y. Dorogovtsev and O. A. Ortega, "On the existence of periodic solutions of a stochastic equation in a Hilbert space," Vīsnik Kiïvs'kogo Unīversitetu, Serīya Matematiki ta Mekhanīki, vol. 115, no. 30, pp. 21-30, 1988.

[6] G. Da Prato and C. Tudor, "Periodic and almost periodic solutions for semilinear stochastic equations," Stochastic Analysis and Applications, vol. 13, no. 1, pp. 13-33, 1995.

[7] C. A. Tudor, "Almost periodic solutions of affine stochastic evolution equations," Stochastics and Stochastics Reports, vol. 38, no. 4, pp. 251-266, 1992.

[8] C. A. Tudor and M. Tudor, "Pseudo almost periodic solutions of some stochastic differential equations," Mathematical Reports (Bucureşti), vol. 1, no. 2, pp. 305-314, 1999.

[9] D. N. Keck and M. A. McKibben, "Functional integrodifferential stochastic evolution equations in Hilbert space," Journal of Applied Mathematics and Stochastic Analysis, vol. 16, no. 2, pp. 141-161, 2003.

[10] D. N. Keck and M. A. McKibben, "Abstract stochastic integrodifferential delay equations," Journal of Applied Mathematics and Stochastic Analysis, no. 3, pp. 275-305, 2005.

[11] J. Liang, T. J. Xiao, and J. van Casteren, "A note on semilinear abstract functional differential and integrodifferential equations with infinite delay," Applied Mathematics Letters, vol. 17, no. 4, pp. 473-477, 2004.

[12] T. J. Xiao and J. Liang, "Blow-up and global existence of solutions to integral equations with infinite delay in Banach spaces," Nonlinear Analysis: Theory, Methods \& Applications, vol. 71, no. 12, pp. e1442-e1447, 2009.

[13] M. M. Fu and Z. X. Liu, "Square-mean almost automorphic solutions for some stochastic differential equations," Proceedings of the American Mathematical Society, vol. 138, no. 10, pp. 3689-3701, 2010.

[14] Y. K. Chang, Z. H. Zhao, and G. M. N'Guerekata, “Squaremean almost automorphic mild solutions to non-autonomous stochastic differential equations in Hilbert spaces," Computers and Mathematics with Applications, vol. 61, no. 2, pp. 384-391, 2011.

[15] P. Acquistapace and B. Terreni, "A unified approach to abstract linear nonautonomous parabolic equations," Rendiconti del Seminario Matematico della Università di Padova, vol. 78, pp. 47-107, 1987.

[16] D. Kannan and A. T. Bharucha-Reid, "On a stochastic integrodifferential evolution equation of Volterra type," Journal of Integral Equations, vol. 10, pp. 351-379, 1985.

[17] Ø. Bernt, Stochastic Differential Equations, Springer, Berlin, Germany, 2005.

[18] T. Diagana and G. M. N'Guérékata, "Almost automorphic solutions to semilinear evolution equations," Functional Differential Equations, vol. 13, no. 2, pp. 195-206, 2006.

[19] T. Diagana and G. M. N'Guérékata, "Almost automorphic solutions to some classes of partial evolution equations," Applied Mathematics Letters, vol. 20, no. 4, pp. 462-466, 2007.

[20] G. M. N'Guerekata, Almost Automorphic and Almost Periodic Functions in Abstract Spaces, Kluwer Academic, New York, NY, USA, 2001.

[21] G. M. N'Guérékata, Topics in Almost Automorphy, Springer, New York, NY, USA, 2005. 
[22] G. Da Prato and J. Zabczyk, Stochastic Equations in Infinite Dimensions, vol. 44 of Encyclopedia of Mathematics and its Applications, Cambridge University Press, Cambridge, UK, 1992.

[23] M. Zaki, "Almost automorphic solutions of certain abstract differential equations," Annali di Matematica Pura ed Applicata, vol. 101, pp. 91-114, 1974.

[24] P. Acquistapace, "Evolution operators and strong solutions of abstract linear parabolic equations," Differential and Integral Equations, vol. 1, no. 4, pp. 433-457, 1988.

[25] A. Lunardi, Analytic Semigroups and Optimal Regularity in Parabolic Problems, vol. 16 of Progress in Nonlinear Differential Equations and Their Applications, Birkhäuser, Basel, Switzerland, 1995.

[26] A. Ichikawa, "Stability of semilinear stochastic evolution equations," Journal of Mathematical Analysis and Applications, vol. 90, no. 1, pp. 12-44, 1982.

[27] X. L. Li and Y. L. Han, "Square-mean almost periodic solutions to some stochastic evolution equations," Acta Mathematica Sinica. In press.

[28] L. Maniar and R. Schnaubelt, "Almost periodicity of inhomogeneous parabolic evolution equations," in Evolution Equations, vol. 234 of Lecture Notes in Pure and Applied Mathematics, pp. 299-318, Marcel Dekker, New York, NY, USA, 2003. 


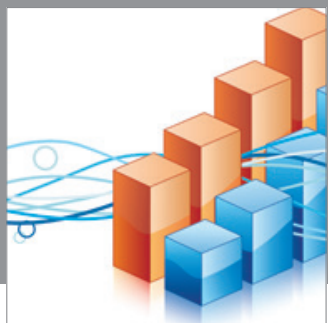

Advances in

Operations Research

mansans

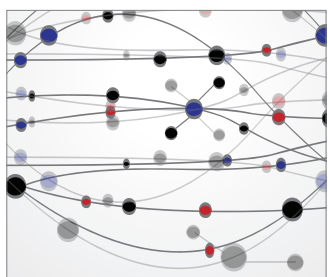

The Scientific World Journal
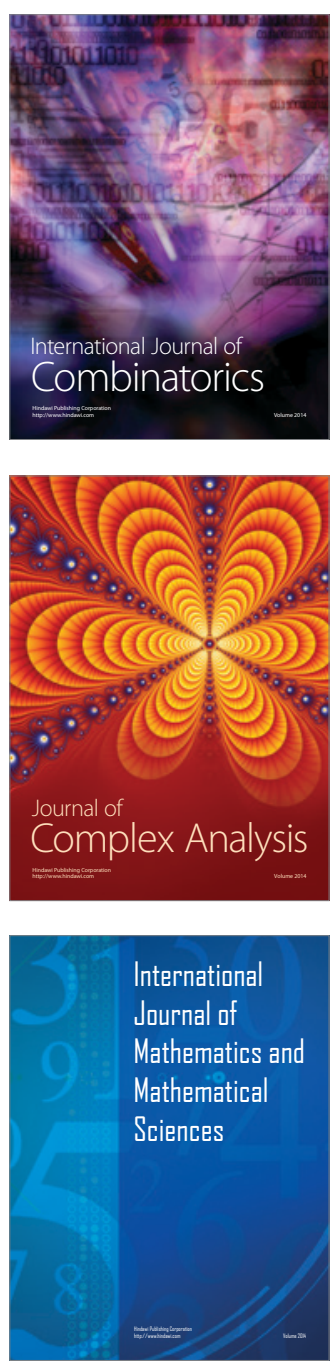
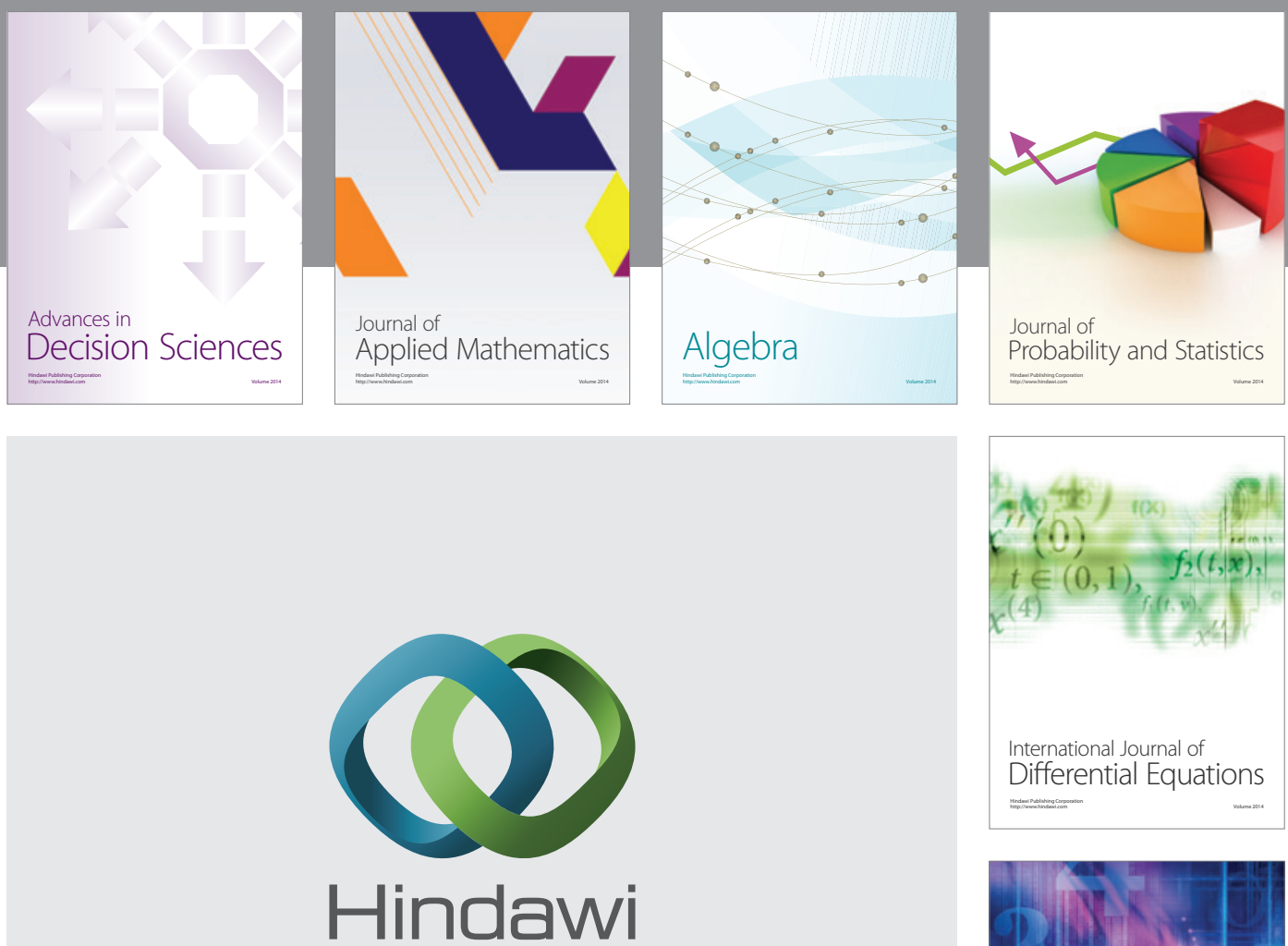

Submit your manuscripts at http://www.hindawi.com
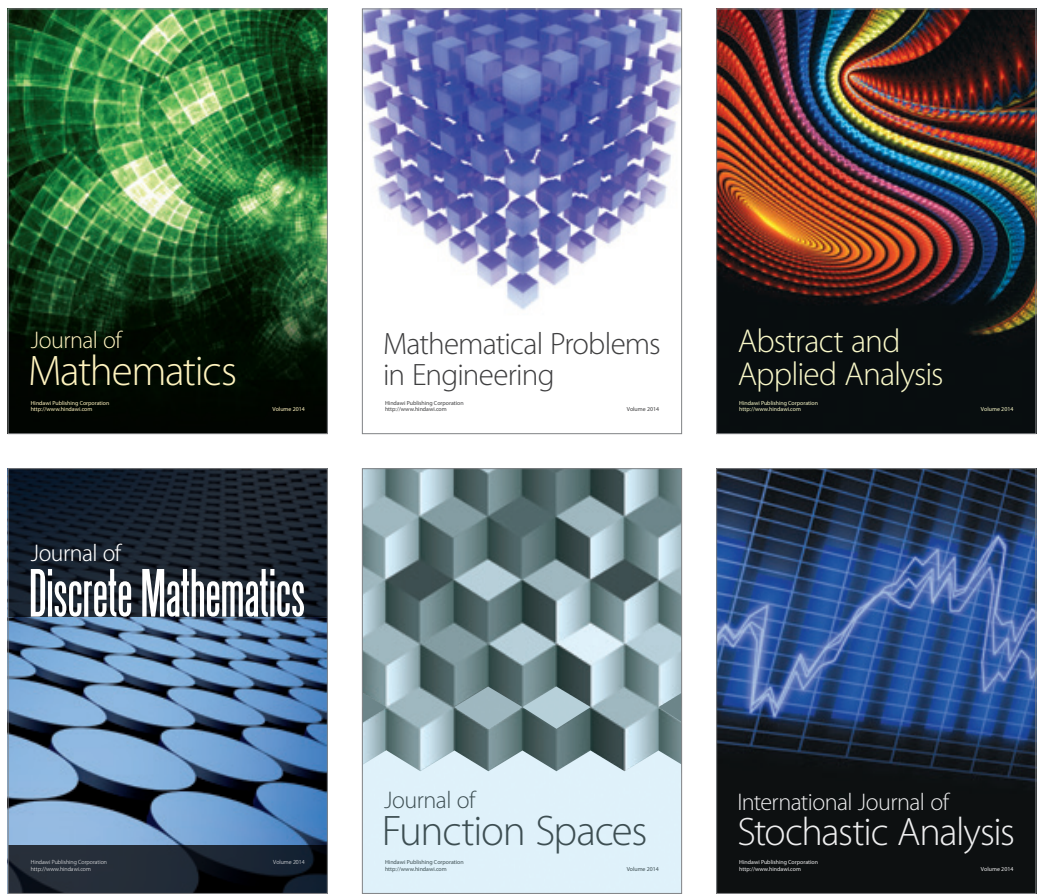

Journal of

Function Spaces

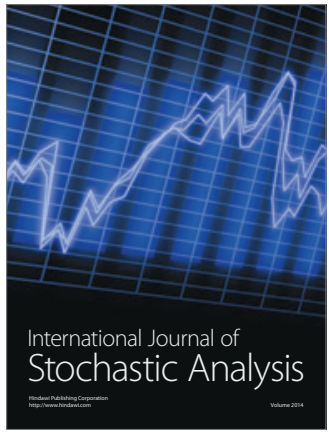

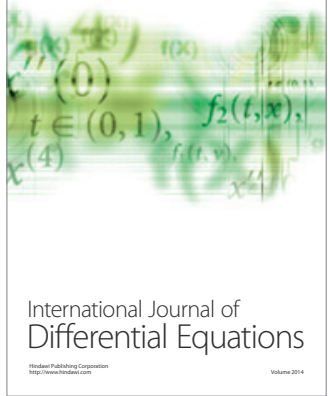
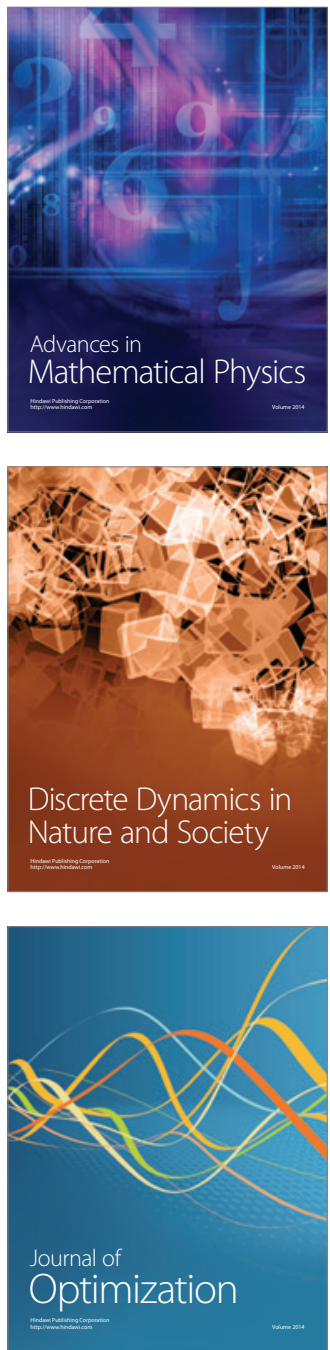